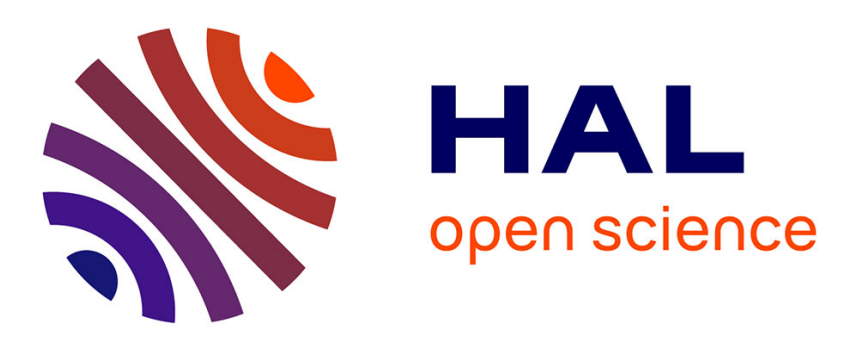

\title{
An Efficient Certificateless Blind Signature Scheme in the Random Oracle Model
}

\author{
Hao Xu, Baoyuan Kang, Yongzheng Niu
}

\section{To cite this version:}

Hao Xu, Baoyuan Kang, Yongzheng Niu. An Efficient Certificateless Blind Signature Scheme in the Random Oracle Model. 11th IFIP International Conference on Network and Parallel Computing (NPC), Sep 2014, Ilan, Taiwan. pp.563-566, 10.1007/978-3-662-44917-2_53 . hal-01403143

\section{HAL Id: hal-01403143 \\ https://hal.inria.fr/hal-01403143}

Submitted on 25 Nov 2016

HAL is a multi-disciplinary open access archive for the deposit and dissemination of scientific research documents, whether they are published or not. The documents may come from teaching and research institutions in France or abroad, or from public or private research centers.
L'archive ouverte pluridisciplinaire HAL, est destinée au dépôt et à la diffusion de documents scientifiques de niveau recherche, publiés ou non, émanant des établissements d'enseignement et de recherche français ou étrangers, des laboratoires publics ou privés. 


\title{
An Efficient Certificateless Blind Signature Scheme in the Random Oracle Model
}

\author{
Hao Xu ${ }^{1, a}$, Baoyuan Kang ${ }^{1, b}$, Yongzheng Niu ${ }^{1, c}$ \\ ${ }^{1}$ School of Computer Science and Software Engineering, Tianjin Polytechnic University, Tianjin, \\ 300387, China \\ a23880797@qq.com, bbaoyuankang@aliyun.com, ${ }^{c}$ niuer890214@163.com
}

\begin{abstract}
The blind signature schemes are useful in some applications where the anonymity is a thorny issue. The certificateless public key cryptography (CLPKC) can eliminate the certificate management problem and solve the key escrow problem. In this paper, we put forward a secure and efficient CLBS scheme. We then illustrate that our new scheme is secure in the random oracle model. Also, we theoretically validate that our proposed scheme is more efficient than those existing ones in terms of computational complexity. We hope to transfer our scheme into applications.
\end{abstract}

Keywords: Certificateless blind signature; Certificateless public key cryptography; Random oracle model; Computational Diffie-Hellman problem

\section{Introduction}

The blind signature was first proposed by Chaum [1], which can provide the anonymity of signed message. Informally, blind signature allows the message owner blind the message by the blind factors, and then lets the signer sign the blinded message. At last, the message owner eliminates the blind factors of signature to get the signer's signature of the original message. Blind signature is a special digital signature, it must also meet the property of blindness differing from other signatures. Therefore, blind signature schemes can used in order to eliminate the possible abuse of linkability.

Up to now, even if there have been a lot of researches for blind signature, most of works have been based on a traditional public key infrastructure (PKI) or an identity-based public key cryptography (ID-PKC). In the traditional PKI, the trusted Certificate Authority (CA) needs a large amount of storage and computing time to manage the certificates, which are signatures of CA on the public keys of users. This is called certificate management problem. In the ID-PKC, an inherent problem of ID-PKC is that a Key Generation Center (KGC) generates any user's private key with a master key of KGC. Obviously, a malicious KGC is able to forge the signature of any signer. This is key escrow problem. To tackle the prob- 
lems above, Al-Riyami and Paterson [2] put forward a new paradigm named certificateless public key cryptography (CL-PKC) in 2003, which avoids the certificate management problem in traditional PKI and eliminates the key escrow problem in ID-PKC.

Blind signature and CL-PKC have gotten fruitful achievements since they were introduced. However, to our best knowledge, little attention has been paid to the design of provably secure blind signature scheme in CL-PKC [3]. In this paper, we propose an efficient CLBS scheme based on bilinear pairings, then show that our CLBS scheme is existentially unforgeable in the random oracle model under the Computational Diffie-Hellman (CDH) problem.

The remainder of this paper is organized as follows: In Section 2, we present the construction of our new CLBS scheme. We will show that our scheme is security in Section 3. Section 4 shows efficiency comparison with the existing schemes.

\section{Certificateless Blind Signature Scheme}

In this section, we propose a secure and efficient CLBS scheme. It consists of the following seven algorithms. The details are shown as follows:

- Setup: On the input of a security parameter $k$, the KGC firstly selects a cyclic additive group $G_{1}$ generated by a generator $P$ of prime order $q$, a cyclic multiplicative group $G_{2}$ with the same order $q$ and a bilinear map $e: G_{1} \times G_{1} \rightarrow G_{2}$, picks the master key master-key $s \in{ }_{R} Z_{q}^{*}$ at random and keeps $s$ secret, then sets $P_{p u b}=s P$ as the public key. Choose three secure hash functions: $H_{1}:\{0,1\}^{*} \rightarrow G_{1}, H_{2}:\{0,1\}^{*} \rightarrow G_{1}, H_{3}:\{0,1\}^{*} \rightarrow Z_{q}^{*}$. The system parameters are params $=\left\{G_{1}, G_{2}, q, e, P, P_{p u b}, H_{1}, H_{2}, H_{3}\right\}$.

- Partial-Private-Key-Extract: For a user with identity $I D_{A} \in\{0,1\}^{*}$, KGC computes $Q_{\mathrm{A}}=H_{1}\left(I D_{A}\right)$ as the public identity of the user, and sends $D_{A}$ to the user as his partial private key via a secure channel, where $D_{A}=s Q_{A}$.

- Set-Secret-Value: Given params, the user with identity $I D_{A}$ selects a random $x_{A} \in{ }_{R} Z_{q}^{*}$ as his secret value.

- Set-Public-Key: This algorithm accepts params, a user's identity $I D_{A}$ and secret value $x_{A}$, then outputs the public key $P_{A}=x_{A} P$ of the user with identity $I D_{A}$.

- Set-Private-Key: This algorithm takes as input params, the signer's identity $I D_{A}$, partial private key $D_{A}$, public key $P_{A}$ and secret value $x_{A}$ to produce the 
signer's private key $S K_{A}=D_{A}+x_{A} T_{A}$, where $T_{A}=H_{2}\left(I D_{A}, P_{A}\right)$.

- Issue: To sign a message $m$, the signer with identity $I D_{A}$, public key $P_{A}$, private key $S K_{A}$, executes the following steps with the signature requester:

(a) Request: The requester requests the signer for a CLBS. After receiving the request, the signer chooses $r \in{ }_{R} Z_{q}^{*}$ at random and computes $R^{\prime}=r P$, then sends $R^{\prime}$ to the requester.

(b) Blind: Upon receiving $R^{\prime}$, the requester randomly picks $\alpha, \beta \in{ }_{R} Z_{q}^{*}$ as the blind factors, computes $R=\alpha R^{\prime}+\beta P, \quad h^{\prime}=H_{3}\left(m, I D_{A}, P_{A}, R\right)$ and $h=\alpha^{-1} h^{\prime}$, then sends $h$ back to the signer.

(c) Sign: The signer sends $S^{\prime}$ to the requester, where $S^{\prime}=h S K_{A}+r P_{p u b}$.

(d) Unblind: The requester unblinds $S^{\prime}$ by computing $S=\alpha S^{\prime}+\beta P_{p u b}$, and outputs $\sigma=(R, S)$ as the CLBS on message $m$.

- Verify: For a message $m$, and the corresponding signature $\sigma=(R, S)$, the verifier computes the value $h^{\prime}=H_{3}\left(m, I D_{A}, P_{A}, R\right), T_{A}=H_{2}\left(I D_{A}, P_{A}\right)$, then check if the equation:

$$
e(S, P)=\left(e\left(Q_{A}, P_{p u b}\right) e\left(T_{A}, P_{A}\right)\right)^{h^{\prime}} e\left(R, P_{p u b}\right)
$$

holds. If the equation holds, the signature $\sigma=(R, S)$ is valid.

\section{Security}

About the security of our CLBS scheme, we have the following two theorems. Theorem 1. The CLBS scheme is blindness.

Theorem 2. The CLBS scheme is existentially unforgeable under assuming that the $\mathrm{CDH}$ problem in a cyclic additive group $G_{1}$ is intractable.

\section{Efficiency Analysis}

We compare our scheme with other three available CLBS schemes [4-6] based on bilinear pairings in terms of secret key size and the required computational cost of signing and verifying. For our scheme, we omit the computation efforts which can be pre-computed by the verifier, for example, the computation of $e\left(Q_{A}, P_{p u b}\right)$ and $e\left(T_{A}, P_{A}\right)$. For convenient comparison, we include the following presentation, the notion $\left|G_{1}\right|$ denotes the bit length of an element in $G_{1},|q|$ 
be the binary length of an element in $Z_{q}, P_{m}$ be the scalar multiplication on the curve, $P_{e x}$ be the exponentiation operator in $G_{2}$ and $P_{e}$ be the bilinear pairing operation.

Table. 1. Performance comparison of different schemes

\begin{tabular}{cccc}
\hline Scheme & Sign & Verify & Secret key length \\
\hline$[4]$ & $3 P_{e}+4 P_{e x}+7 P_{m}$ & $1 P_{e}+1 P_{e x}+2 P_{m}$ & $|q|+\left|G_{1}\right|$ \\
{$[5]$} & $2 P_{e}+1 P_{e x}+8 P_{m}$ & $3 P_{e}+1 P_{e x}+2 P_{m}$ & $|q|+\left|G_{1}\right|$ \\
{$[6]$} & $3 P_{e}+2 P_{e x}+9 P_{m}$ & $2 P_{e}+1 P_{e x}$ & $|q|+\left|G_{1}\right|$ \\
Our scheme & $7 P_{m}$ & $2 P_{e}+1 P_{e x}$ & $\left|G_{1}\right|$ \\
\hline
\end{tabular}

From Table 1, we can clearly see that a prominent merit in our scheme is that no pairing operator is required in the whole signing process. To our best knowledge the computation of the pairing is the most time-consuming in pairing based cryptosystem. Besides, the length of the secret key is also shorter than other schemes. Thus, our scheme is more useful and efficient than the previous schemes.

\section{Conclusion}

In this paper, we put forward a new CLBS scheme on the bilinear pairings, and give some theorems of the security and efficiency analysis of our scheme, which show that the new proposed CLBS scheme is much more efficient and satisfy both blindness and unforgeability properties. Our CLBS scheme may have applications in areas such as electronic cash systems using CL-PKC.

\section{References}

1. Chaum, D.: Blind Signatures for Untraceable Payments. In: Crypto. 1982, pp. 199-203.

2. Al-Riyami, S.S., Paterson, K.G.: Certificateless Public Key Cryptography. In: Advances in Cryptology-ASIACRYPT 2003, pp. 452-473. Springer, Heidelberg (2003)

3. Zhang, L., Zhang, F.T., Qin, B., Liu, S.B.: Provably-secure Electronic Cash Based on Certificateless Partially-blind Signatures. In: Electronic Commerce Research and Applications, 10 (5) (2011), pp. 545-552.

4. Zhang L, Zhang F. Certificateless Signature and Blind Signature[J]. Journal of Electronics (China), 2008, 25(5): 629-635.

5. Zhang L, Zhang F, Qin B, et al. Provably-secure Electronic Cash Based on Certificateless Partially-blind Signatures[J]. Electronic Commerce Research and Applications, 2011, 10(5): 545-552.

6. Liu J, Zhang Z, Sun R, et al. Certificateless Partially Blind Signature[C]//Ad- vanced Information Networking and Applications Workshops (WAINA), 2012 26th International Conference on. IEEE, 2012: 128-133. 